\title{
INTEGRATED APPLICATION FRAMEWORK FOR LOCATION-BASED HEALTH INFORMATION SERVICES
}

\author{
Đorđe Vukelić, Verka Jovanović \\ Singidunum University, Belgrade, Serbia
}

\begin{abstract}
:
Location based services are one of the leading trends in the new developments of the information technology. Their impact could be seen in the different sectors of society, especially in the health sector. Very closely connected to the new paradigm of mobility, detecting the patient locations, especially in the relation of the different decision in public health is becoming one of the most important point in location-based health information services. Contrary to isolated use of GPS and/or GIS in specific issues related with patient locations and impact to different questions/areas in public health, there is a need to formulate the integrated approach. Positioning/locating technologies with the communications networks and GIS as synergy integrator would be the base to formulate the new framework of this integrated approach.
\end{abstract}

\section{Key words:}

GIS,

GPS,

location,

health,

integration,

framework.

\section{INTRODUCTION}

Historically, it is well known in medicine that location can influence health. Location, as a concrete "expression or concretization" of the space, plays a key role in determining the type and nature of human activity. In fact, different places on Earth are usually associated with different profiles that can also change with time: physical, biological, environmental, economic, linguistic, social, cultural, and sometimes even spiritual profiles, that do affect and are affected by health, disease, and healthcare [1].

The world is changing from the industrial age to the information age. The information technology offers a wealth of health information resources that can answer most of the knowledge needs of clinicians and their patients, and the public in general, but also carries with it the risk of overloading them with unnecessary information [2].

However, the rise of information technology marks a more complex relations between space and time. The accent must be on the approach to treat the space and time as the key, integrated entities in the "new mobility". In that respect, Geographic information system (GIS) as a tool is powerful means of converting spatially collected data into information and combining them with attribute type information. The functionality for such systems included the ability to geo-code patient data and push-pin patients and health services.
A key challenge remains to find and define integrated framework which could be used in the different aspects of location-specific knowledge to patient (users) based on their location but at the same time could be applied in the relation with different areas of public health.

\section{USABILITY OF LOCATION-BASED SERVICES IN HEALTH AND HEALTHCARE}

\section{Location-based services supported by technological mobility}

Location based services are not merely about location, in this sense, and can be read as technologies of mobility. As Cresswell [3] writes, mobility is "the entanglement of movement, representation, and practice". In this sense, mobilities demand the recognition of the spatiality of our worlded interactions, despite the ways in which space and time are "tamed", to use Massey [4], by some methods for measuring movement. The rise of information technology marks a further enabling of the turning of space into time, while also producing more complex relationships of mobility between the here and there. These geographies of information technology emphas-ize the ways in which technology imbricates in everyday life.

State-of-the-art mobile technology has evolved from the simple pagers and bulky mobile phones of yesterday to 
to-day's multifunctional smartphones and computer tablets. This modern mobile landscape is supported by digital networks that are available around the globe.

As location-based services are becoming increasingly part of the smart phones, what is the impact in healthcare?

According to a survey conducted in August-September $2012,31 \%$ of smart phone owners say they use their phone to look for health or medical information online. That is up from 17\% of smart phone owners in September 2010. (Source Pew Internet/CHCF Health Survey) [5].

\section{Location-specific health information}

The location-specific health information:

- local disease rates, maps and guidelines

- targeted health education

- addresses of local healthcare facilities

- local health news

- local weather, pollen and air quality alerts and maps

- local health risks and hazards

- travelers' health information

- local drugs/ drug trade names and prices

- information whose digital distribution rights are limited to some location(s)

In more descriptive mode, location based healthcare services could be used to find the nearest and most accessible hospitals or clinics provided by an online healthcare facility locator service based on their location and health needs, and even provide them with driving directions and real-time traffic information. Ambulance and rescue teams can quickly respond and precisely track people in need of a medical emergency. It can also send cautions or alarm messages to mobile patients for environmentally irritated or polluted areas [6]

However, currently, there are issues and limitations of the location-based services in health and healthcare:

1) There is no technology integration. Successful large-scale deployment of location based services will require a blend of competencies. A key driver of location-based-services will be a degree to which there is a fit between the system's technical feasibility and the overall health strategy guiding its usage. These multiple technologies and platforms (including portable digital assistants, mobile phones, gaming devices, etc.) need to be globally connected and integrated with the wireless network infrastructure. Software innovations which are meant for designing low-cost reliability and quality from such a complex puzzle of disparate software, hardware, and connectivity components will accelerate networking effects that, in turn, enables the technology integration.

2) Mainly based on GPS technology to give the location. Failure to establish a GPS fix in difficult environments such as urban canyons/foliage/coated windshield environments, and indoors/inside buildings. Also, location-based services must take into consideration the input and output character- istics of different user devices by carefully choosing, personalizing and formatting the content to display on such devices [7].

3) Privacy issues. Location capability poses service providers with the challenge of responsibly handling patient' per-sonal privacy [8]. This is particularly important with tracking services that continuously monitor user's location.

\section{THE COMPONENTS OF INTEGRATED APPLICATION FRAMEWORK FOR LOCATION- BASED HEALTH INFORMATION SERVICES}

\section{Technological feasibility and socially acceptable options of GIS}

Geographic information systems (GIS) followed the development of information system. In the early days, GIS was mainly the "repository" of information. Today, GIS is the tool to collect, analyze and present the information where the accent is on analyzing. GIS is becoming very important modeling tool, where the models are not only based on quantitative, but includes elusive measures. These are not the usual quantitative measures amenable to computer algorithms and traditional decision-making models [9].

It is important to highlight that GIS bridges the gap between technically possible to socially acceptable solutions. The visual presentation, most often map, as a result of "if what" scenario are possible option or solutions. This realization, coupled with active involvement in the decision process, can lead to group consensus [10].

Comparing to other types of information systems, GIS is always using holistic approach. Related by accurate spatial information, an incredible variety of real-world and projected past or future data can be analyzed, interpreted and represented to facilitate education and decision making [11].

\section{Combined approach - outline of the new framework}

The new framework with the holistic approach would be encapsulating the following:

- Positioning/locating technologies integrated with the communications networks

- GIS - the synergy integrator

The integration of the positioning/locating technologies with the communications networks would need to combine the following technologies:

- GPS

- Cell tower triangulation

- A-GPS

- Cell ID

- WPS

- Browser-based location

GPS still remains the most popular and most widely spread positioning technology commercially available to- 
day. However, GPS technology has several serious drawback of which the critical that is not working in the most indoor or covered environments.

Cell tower triangulation uses the known speed of radio signals (constantly emitted by the mobile phone on UHF fre-quencies) to calculate the distance from receivers. Because cell tower triangulation is a network-based lo-calization technique, it requires an agreement with the mobile operator in order to adopt it within a mobile service.

Fundamentally, A-GPS tries to address the key inescapable drawback of GPS technology, namely, that a location fix is impossible in most indoor or covered environments.

Cell ID has gained significantly in popularity as a positioning method in the last few years. Cell ID positioning is accomplished by using the serving cell tower (the tower that a mobile device is communicating with), or the cell, and its known position to find the mobile device's position.

A key advantage of WPS, indicating they are a musthave for many mobile applications, is that they work indoors where traditionally GPS hasn't been available.

Location-based services are no longer limited to mobile or GPS devices. Web services running in browsers can now access a user's location through IP geocoding or centralized databases.

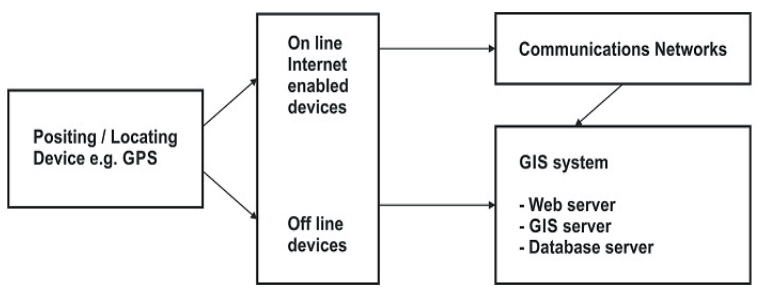

Figure 1. Positioning/locating technologies integrated with the communications networks, Source: Authors

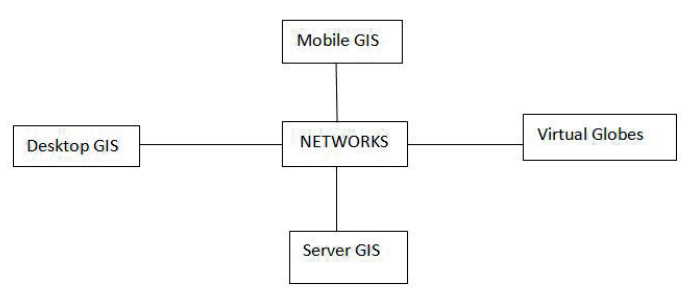

Figure 2. GIS - The Synegry integrator, Source: Authors

The previous section with the overview of the locating/positioning technologies clearly indicates the different categories of the location information. A descriptive location is always related to natural geographic objects like territories, mountains, and lakes, or to man-made geographical objects like borders, cities, countries, roads, buildings, and rooms within a building. A spatial location represents a single point in the Euclidean space. It is usually expressed by means of two- or three-dimensional coordinates, which are given as a vector of numbers, each of it fixing the position in one dimen-sion. Topology of a communications network, for example, the Internet or cellular systems like GSM refer to the network locations.
Hence, an important function of new the framework is the integration (or maybe better say translation) between the different categories of locations. If positioning delivers a spatial or network location, it must often be mapped onto a descriptive location in order to be interpretable by the respective user.

Geographic Information System (GIS) is the essential key technologies for fulfilling these tasks. GIS, comparing to "classical" information systems, has unique features, which enables the integration of the different components these fea-ture are:

- Underlying levels of abstraction

- Map Analysis

- Modeling

- Visualization

The upper layer in a GIS, the so-called geographic data model, provides a conceptual view of geographic content in terms of units called features. A feature represents a real-world entity, for example, a building, road, river, country, or city. The lower layer consists of a spatial component, which fixes its location, shape, and topological relationship with oth-er entities, and a description, which provides non-spatial information about the entity, for example, the name of a city or road, or the population of a country. The relation, real-world entity=spatial component+non-spatial information, enables GIS to be implemented in the different forms and the different level.

The new trends in the main areas of technology confirms the previous concept.

Launched in November 2010, Kinect is a motion sensing USB (Universal Serial Bus) input device by Microsoft that enables users to control and naturally interact with games and other programs without the need to physically touch a game controller or object of any kind. Kinect achieves this through a natural user interface by tracking the user's body movement and by using gestures and spoken commands [12].

The hands-free convenience of gesture and speech recognition can prove extremely useful in a number of practical and exclusive 'use scenarios' where mouse or multi-touch screen inputs are difficult, such as when delivering presentations involving 3-D virtual globes on a large screen on stage to a large audience, e.g., this presentation of Microsoft's WorldWide Telescope using the Kinect sensor: [13].

Collaborative GIS in virtual situation rooms involving distributed teams of users [14] is another 'use scenario' that can benefit from Kinect's unique features such as its headset-free 3-D, in addition to its use as 3-D motion sensing/gestures and speech recognition NUI for controlling a shared 3-D virtual globe during networked spatial data presentations. Using the Kinect sensor to create 3-D maps of real world locations and objects $[15,16]$ is still not very refined for serious use, but might soon get added to the GIS professional's toolkit as the technology evolves and matures. 


\section{APPLYING INTEGRATED FRAMEWORK PROJECT PHARMACY OF YOUR CHOICE (POYC) MINISTRY OF HEALTH (REPUBLIC OF MALTA)}

Malta is a southern European country in the Mediterranean Sea. With the area of $316 \mathrm{~km} 2$ and the population of 400000 people, Malta is one of the world's smallest and most densely populated countries. Malta's population density of 1,282 per square $\mathrm{km}$ is by far the highest in the EU, and one of the highest in the world [17].

Very limited space with population density and advance economy has a serious impact in many different sectors. One of this area is public health.

Historically, most of the functions in the public health are centralized. Previously, St. Luke's hospital and from 2007 Mater Dei Hospital is the main hospital in Malta. One of very important functionality of public health is distribution of pharmaceutical products

The initiative of Government of Malta to provide free pharmaceutical products is regulated by the Social Security Act (1987). Forming part of this Act is Schedule Five, which specifically outlines the diseases and conditions for which free medical care is to be accorded. The specified ailments in this compilation are all chronic conditions (from which affected individuals can never fully recover, but can only medicate themselves to reduce the condition's effects on their health) and medical remedies are to be provided by Government solely on the presence of any of the specified conditions to all affected individuals, irrespective of any other factors such as age or income.

Prior to the introduction of the Pharmacy of your Choice (POYC) Scheme, the dispensing of these free pharmaceutical products was carried out by the Government pharmacies situated in five health centres and hospitals across Malta and Gozo. The few locations available for the collection of these pharmaceutical products meant that a large concentration of clients would amass at each particular point, inevitably creating lengthy queues. The small number of locations from which these pharmaceuticals were dispensed also meant that individuals entitled to use this system of dispensation had to generally travel further than walking distance to collect their entitlements, thereby adding to the overall inconvenience of the experience.

It was necessary to do better redistribution in spacetime formula. The first geospatial analysis using GIS as main tool, clearly shows the discrepancy of the number of patient and the health centres which very used for the distribution of the free pharmaceutical products. Not only, there was very high ratio between the areas from where the patients are living and the location where they collects the free pharmaceutical products, it was very evident that every month this "image" changes. As well, in a view of "old habits" very high percentage of the patients were still coming to the main hospital inevitably creating lengthy queues and making even more problems regarding the parking problems for other patients/visitors of main hospital.

Comparing the number of the people entitled for the free pharmaceutical products and their place of living it was very clear that the only solution has to be multi centers in the same location of patients living or working space. Considering that building or opening new government centers will be very expensive, the small pilot project was launched. The idea behind this project was to analyze the locations of private pharmacies and locations of patients (living or working place). As a conclusion, this project would give the answer of the possibilities to use private pharmacies as the distribution points of the free pharmaceutical products.

To make the analysis possible the GPS was used to take the coordinates of each pharmacy (in the area of pilot project) and the patients who were chosen.

Application GPS_MT was developed for the smart phones with the following functions:

- Using embedded GPS device

- Send GPS coordinates to server with SMS or Email

- Integration with Google maps

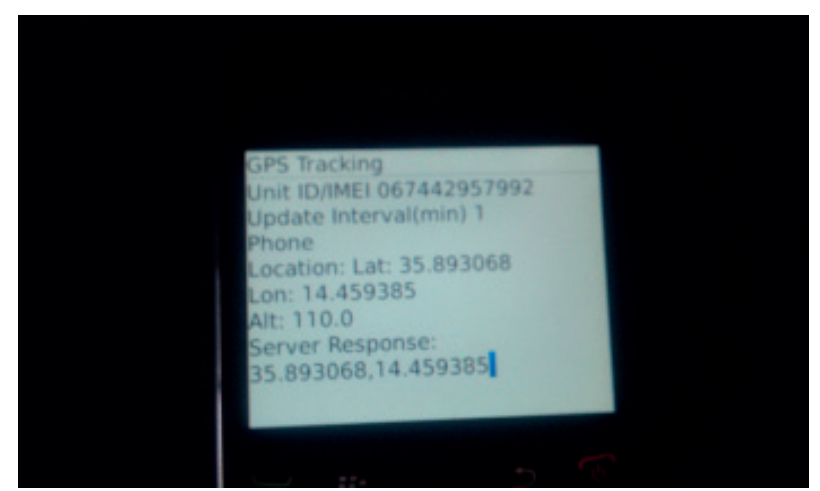

Figure 3. GPS_MT application for smart mobile phone, Source: Authors

Comparing the locations of the patient and private pharmacies, it was very clear that the network of private pharmacies in local council would be sufficient model for space organization of distribution of free pharmaceutical products.

In view of previously mentioned shortcomings and the results of GIS initial analysis, Government of Malta saw fit to implement a system whereby dispensation of pharmaceutical products would be carried out through private pharmacies. Under such a Scheme, patients would be able to collect their entitlement at a private pharmacy of their choice and convenience. This meant that the dispensing points would, as a result, greatly multiply, an intended effect central to addressing the problems of congestion and travelling distance prevalent in the old system.

In July 2007, an agreement was therefore signed between the Ministry of Health, the Elderly and Community Care (MHEC), the Ministry of Finance, the Economy and Investment, the Malta Chamber of Pharmacists and the Pharmacy Section of the Chamber of Small and Medium Enterprises within the General Retailers and Traders Union, through which the POYC Scheme was initiated. The POYC Unit was also set up and given the mandate to implement and manage this new client-centric system. The overall objectives of this Unit are to facilitate a more comfortable access to the Government's free pharmaceutical 
service by shifting this service from the Health Centres and moving it closer to their (the beneficiaries') place of residence - to any pharmacy of the patients' own choice [18].

Launching the project, one of the most important think was to have in place very reliable and secure information system. It was necessary to have the system in place which will be able to do:

- encompasses stock movement

- commencing at the stock acquisition stage,

- addressing storage and processing issues,

- delivery to the participating pharmacies,

- delivery to the patient.

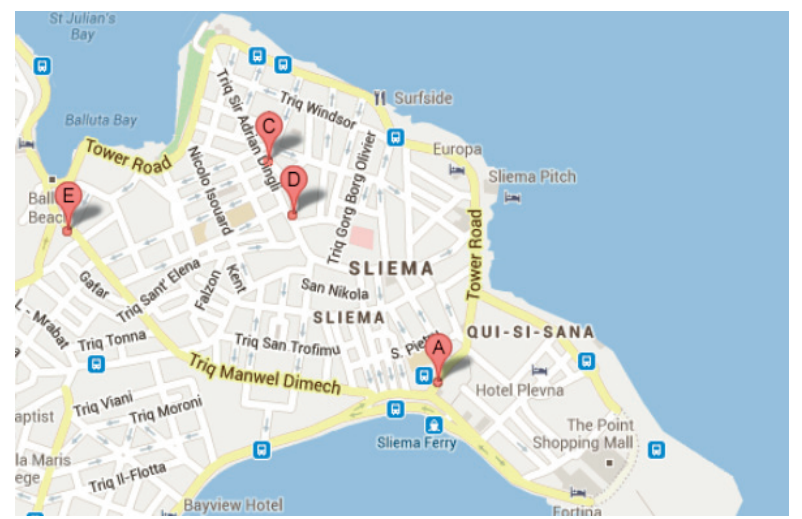

Figure 4. Distribution of local pharmacies in local council Sliema, Source Ministry of Health, Malta, Source: Authors

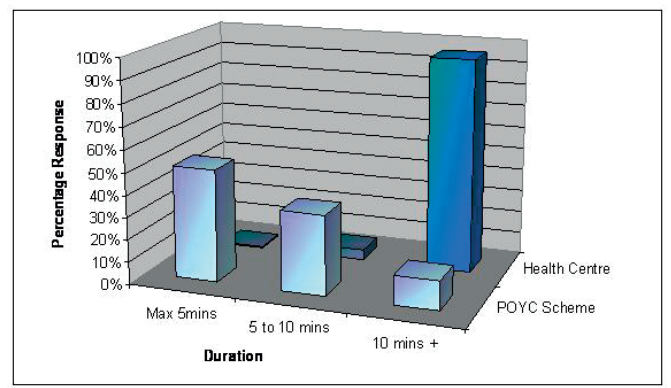

Figure 5. Comparison of Perceived Waiting Times of POYC Scheme and Health Centre Distribution, National Audit Office, Survey January, 2012, Source: Authors

Furthermore, during the development, the POYC Unit highlighted the fact that the IT interface must be very secure. This was achieved through a series of access controls, effectively limiting which pharmaceutical products could be dis-pensed by pharmacists to beneficiaries. Dispensation was thereby limited to the specific condition/s the end client was registered for on Schedule Five. It was explained that once the pharmacist accessed the respective beneficiary's profile, the system only allowed dispensation of pharmaceutical products which corresponded to the ailment/s for which the particular beneficiary was registered. Another important consideration in this regard is that as opposed to the type of dispensed pharmaceuticals, the quantities of the dispensed entitlement were subject to the alteration by the dispensing pharmacist, in accordance with the doctor's prescription.

Considering the nature of this type of the information (patent record, details, medicines etc), one of the most im- portant function of the system was to be able to be used only once the computer is in pharmacy and connected with the main POYS Unit. The original algorithm was developed, based on IP geocoding and the network card of the computer used in the pharmacy.

Due the complexity and nature of the project, POYC was completed last year.

The main results of the system which was combing is the patient oriented needs with public health services, could be seen from the chart Figure 5. It is evident the new space organization of delivering free pharmaceutical products has the major percentage of just max 5 minutes service, where the previous one (centralized in few health centers) has the majority of over 10 minutes.

It is one of the first projects on the national level, which clearly indicated the importance of using integrated framework.

\section{CONCLUSIONS AND RECOMMENDATIONS}

The new trends and developments of the information technology have very important or even the critical impact to all spheres of today's society. Technological mobility, the characteristic of modern world, is changing the traditional meaning of the location and it is opening new horizons in the location based services. Isolated services, which were mainly used to determinate the static type of location/position, will not be sufficient.

All above-mentioned effects are shaping and changing location-based health information services. Detect patient location and understand the value of it in the in the relation with different areas of public health is one of the pre-requisites in the modern health information services.

The value of the new framework of integrated approach will be, exactly, in combining other positioning/ locating technologies with the communications networks where GIS will be synergy integrator on the different levels.

The new research and recommendations will be to create the other prototypes of the software solutions based on the above-mentioned integrated approach.

\section{REFERENCES}

[1] Kamel Boulos MN, Roudsari AV, Carson ER. Health Geomatics: "An Enabling Suite of Technologies in Health and Healthcare” (Methodolical Review). J Biomed Inform. 2001.

[2] Brennan PF, Friede A,. "Public Health and Consumer Uses of Health Information.” In: Shortliffe EH, Perreault LE, editor. In Medical Informatics - Computer Applications in health Care and Biomedicine. New York, Springer-Verlag; 2001.

[3] Cresswell, T., "Towards a politics of mobility”, Environment and Planning D: Society and Space, 2010.

[4] Massey, D.B., "For Space”, London. Sage, 2005.

[5] Pew Internet/CHCF Health Survey, 7 of August - 6 of September 2012 [Online]. Available: [http://pewinternet. org/Reports/2012/Mobile-Health/Main-Findings/MobileHealth.aspx accessed 1 November 2013] 
[6] Wang S, Min JW and Yi BK, "Location Based Services for Mobiles: Technologies and Standards", IEEE International Conference on Communication (ICC) 2008, Beijing, China, 2008.

[7] Leeuwen A., "Geo-targeting on IP Address - Pinpointing Geo-location of Internet Users", GeoInformatics. [Online]. Available: [http://www.geoinformatics.com/issueonline/issues/2001/07_2001/pdf_07_2001/28_31_iptar.pdf ] , 2001

[8] Hogeweg M., Relocation Based Services. GeoInformatics. [Online]. Available: [http://www.geoinformatics.com/issueonline/issues/2001/09_2001/pdf_09_2001/13_hogeweg. pdf ], 2001.

[9] Berry J. A., "Brief history and probable future of geotechnology”, University of Denver, [Online]. Available: [http:// www.innovativegis.com/basis/Papers/Other/Geotechnology/Geotechnology_history_future.htm], 2010.

[10] Berry J., "GIS Evolution and Future Trends", Beyond Mapping III, Compilation of Beyond Mapping columns appearing in GeoWorld magazine 1996 to [Online]. Available: [http://www.innovativegis.com/basis/MapAnalysis/ Topic27/Topic27.htm\#Top], 2013.

[11] Rafi M,. [Online]. Available: http://en.wikipedia.org/wiki/ User:Rafi.km, 2013.

[12] Maged N, Bryan J, Cory Walker, Julio Montero, Aalap Tripathy and Ricardo Gutierrez-Osuna., "Web GIS in prac-tice X: a Microsoft Kinect natural user interface for Google Earth navigation", International Journal of Health Geographics, [Online]. Available: [http://www.ij-healthgeographics.com/content/10/1/45], 2011.
[13] Hollister S., "Microsoft's Kinect navigates the universe thanks to Win-dows SDK" [Online]. Available: [http:// www.engadget.com/2011/04/13/microsofts-kinect-navigates-the-universe-thanks-to-windows-sdk/], 2011.

[14] Kamel Boulos MN., "Novel Emergency/Public Health Situation Rooms and More Using 4-D GIS”. Proceedings of ISPRS WG IV/4 International Workshop on Virtual Changing Globe for Visualisation \& Analysis (VCGVA2009): 27-28; Wuhan University, Wuhan, Hubei, China 2009 (ISPRS Arc-hives, Volume XXXVIII, Part 4/W10, ISSN No: 1682-1777) [Online]. Available [http://www.isprs.org/proceedings/XXXVIII/4-W10/papers/VCGVA2009_03608_ Boulos.pdf], 2009.

[15] Szarski M., "Real World Mapping with the Kinect" . [Online]. Available [https://github.com/mszarski/KinectMapper], 2011.

[16] Brown M, "Kinect hack builds 3D maps of the real world". [Online]. Available [http://www.wired.co.uk/news/ archive/2011-01/24/3d-kinectmap], 2011.

[17] [Online]. Available: http://mt.wikipedia.org/wiki/Malt, 2014.

[18] Ministry of Health, Republic of Malta, [Online]. Available: [https://ehealth.gov.mt/HealthPortal/default.aspx], 2014. 